\title{
Acquisition and clearance of multidrug resistant Acinetobacter baumannii on healthy young adults concurrently burned in a dust explosion in Taiwan: the implication for antimicrobial stewardship
}

Po-Yen Huang ${ }^{1,2 \dagger}$, Shian-Sen Shie ${ }^{1 \dagger}$, Jung-Jr Ye ${ }^{1}$, Shih-Pin Lin ${ }^{3,4}$, Tsui-Ping Liü ${ }^{2,5}$, Ting-Shu Wu ${ }^{1,2}$, Tsu-Lan Wu', Shiow-Shuh Chuang ${ }^{6}$, Ming-Huei Cheng ${ }^{4}$, Yu-Chia Hsieh $^{7^{*}}$ and Ching-Tai Huang ${ }^{1 *}$

\begin{abstract}
Background: Information is limited about the effect of restricted carbapenem use on clearance of multi-drug resistant Acinetobacter baumannii (MDRAB). We sought to determine the time effect of antibiotic exposure on multi-drug resistant Acinetobacter baumannii (MDRAB) acquisition and clearance.
\end{abstract}

Methods: We conducted a retrospective observational study at the intensive care units of a tertiary medical center. Forty-two of a cohort of previously healthy young adults who were concurrently burned by a dust explosion was included. Cases consisted of those from whom MDRAB was isolated during hospitalization. Controls consisted of patients from whom MDRAB was not isolated in the same period. Use of antimicrobial agents was compared based on days of therapy per 1,000 patient-days (DOT/1,000PD). A 2-state Markov multi-state model was used to estimate the risk of acquisition and clearance of MDRAB.

Results: MDRAB was discovered in 9/42 (21.4\%) individuals. The cases had significantly higher use of carbapenem (652 DOT/1,000PD vs. 385 DOT/1,000PD, $P<0.001$ ) before MDRAB isolation. For the cases, clearance of MDRAB was associated with lower use of carbapenem (469 DOT/1,000PD vs. 708 DOT/1,000PD, $P=0.003$ ) and higher use of non-carbapenem beta-lactam (612 DOT/1,000PD vs. 246 DOT/1,000PD, $P<0.001$ ). In multi-state model, each additional DOT of carbapenem increased the hazard of acquiring MDRAB (hazard ratio (HR), 1.08; $95 \%$ confidence interval (CI) 1.01-1.16) and each additional DOT of non-carbapenem beta-lactam increased the protection of clearing MDRAB (HR, 1.25; 95\% Cl 1.07-1.46).

Conclusions: Both acquisition and clearance of MDRAB were related to antibiotic exposure in a homogeneous population. Our findings suggest that early discontinuation of carbapenem could be an effective measure in antibiotic stewardship for the control of MDRAB spreading.

Keywords: Acinetobacter Baumannii, Burn patients, Antibiotic resistance, Antibiotic stewardship, Carbapenem

\footnotetext{
*Correspondence: yuchiahsieh@gmail.com; chingtaihuang@gmail.com

The results of this work were presented orally in part at the American

Society for Microbiology (ASM) Microbe 2016

${ }^{\dagger}$ Equal contributors

${ }^{7}$ Division of Pediatric Infectious Diseases, Department of Pediatrics, Chang

Gung Children's Hospital, Chang Gung University College of Medicine, 5

Fu-Shin St., Kweishan, 333 Taoyuan, Taiwan

'Division of Infectious Diseases, Department of Medicine, Chang Gung

Memorial Hospital and Chang Gung University, 5 Fu-Shin St., Kweishan, 333

Taoyuan, Taiwan

Full list of author information is available at the end of the article
} 


\section{Background}

Acinetobacter baumannii is an important pathogen, causing healthcare-associated infections such as pneumonia, urinary tract infection, soft tissue infection, and bloodstream infection [1, 2]. The extraordinary metabolic versatility of $A$. baumannii contributes to this organism's survival in the environment, including persistence for extended periods of time on dry surfaces [3]. Given this organism's propensity to horizontally acquire resistance to multiple classes of antimicrobial agents, A. baumannii ranks among the most important nosocomial pathogens [4]. The increasing spread of international clones of multidrug-resistant Acinetobacter baumannii (MDRAB) with decreased susceptibility to carbapenem poses a great threat to the health care system; alternative therapeutic options are limited and outcome is poor in patients infected by beta-lactam-resistant $A$. baumannii $[5,6]$. An integrated, multidisciplinary approach is advocated to control the growing threat of MDRAB-related infection or colonization. Proposed responses have included changes in hand hygiene, surveillance, cohort policy, environmental disinfection and cleaning, contact isolation, decolonization, the use of chlorhexidine baths, and antibiotic stewardship programs (ASPs), all with the intent of eliminating this bacterium's reservoir, transmission, and source [7-10].

Studies have shown that antimicrobial use is associated with the emergence of drug-resistant pathogens; the implementation of an ASP may be effective in reducing resistance rates [11-17]. While there is increasing evidence that MDRAB acquisition may be related to prior carbapenem exposure [18-20], there is typically a time lag of one to several months between antimicrobial prescription and the emergence of bacterial resistance $[16,21]$. The most affected patients are those of advanced age, immunosuppressed status, and/or comorbidities that create a need for broader antimicrobial coverage in clinical situations. However, the direct effect of restricted use of carbapenem at the individual patientlevel remains poorly understood.

On June 27, 2015, a dust explosion occurred in the late evening at the Formosa Fun Coast water park in northern Taiwan, creating over 400 burn victims and representing a major challenge to the health care system [22]. Among the victims were forty-two previously healthy young adults who presented with severe burns and were admitted as a cohort to two intensive care units (ICUs) at Chang Gung Memorial Hospital. The dust explosion was caused by a flammable starch-based colored powder. The powder created an extremely dense dust cloud, which immediately caused partygoers to be engulfed by flames when the powder ignited accidentally [23]. This event therefore provided with an opportunity to study the effect of exposure to various antibiotics on the acquisition and clearance of MDRAB in a homogeneous group of patients.

\section{Methods \\ Setting, study design, and the patients}

This case-control study was conducted at the Chang Gung Memorial Hospital-Linkou Branch, a 3700-bed tertiary-care medical center with 308 ICU beds for critical care in northern Taiwan. The facility has two ICUs (48 beds) which were devoted exclusively to care of critically ill burn patients. The present investigation included 42 previously healthy young adults who had been admitted to the ICUs between June 27, 2015 and July 1, 2015 due to the dust burn occurred on June 27, 2015 in Taiwan. This analysis included all inpatient data until discharge or death. The study was approved by the Institutional Review Board of Chang Gung Memorial Hospital (104-9356B) and informed consent was waived due to the study's retrospective nature.

The key managements for the flame burn were accomplished as previously described [23]. In addition, microbiological surveillance was performed during hospitalization. All these burned young adults underwent wound cultures twice a week, tissue cultures whenever surgical debridement was performed. Blood cultures or urine cultures were done if fever and/or sepsis was suspected. Central venous catheter $(\mathrm{CVC})$ would be removed and culture from a CVC tip was performed whenever CVC related infection was suspected. Standard precautions and contact precautions were applied to every patient. Aprons and gloves were used regularly. For appropriate use of antimicrobial agents, a comprehensive ASP has implemented since 2005 at the hospital, with satisfactory outcomes $[13,15]$. The stewardship program involves multidisciplinary professionals including physicians, pharmacist, infection control nurses, as well as policy makers. It is a web-based healthcare information system with prospective audit and online feedback. Both the infectious disease physicians and the pharmacists will review the antimicrobial prescriptions within $48 \mathrm{~h}$. Cases were defined as those from whom MDRAB was isolated from any site during hospitalization. Controls were defined as patients from whom MDRAB was not isolated during hospitalization. For the cases, clearance of MDRAB was defined as two consecutive surveillance cultures of colonized/infected sites negative for MDRAB.

\section{Differentiation between colonization and infection}

Initially we tried to distinguish between infection and colonization status of the patients. However, after reviewing the charts, we found that we could not clearly define infection or colonization status regarding isolation of MDRAB based on objective data. The patients shared similar clinical findings, laboratory data, and isolates that 
were collected intraoperatively. Thus, we defined the condition of the patients as MDRAB infection/colonization.

\section{Data collection and measurement of antimicrobial use} Demographics, underlying diseases, ventilator use, inhalation injury, and catheter use were reviewed. For evaluation of disease severity, an Acute Physiology And Chronic Health Evaluation (APACHE) II score was determined on the first day of admission. To quantify antimicrobial use, the prescriptions of each patient was reviewed and converted into days of therapy (DOTs). We defined the DOT as previously described [24]. In short, one DOT represents the administration of a single agent on a day, without consideration of the number of doses or the strength of dosage. Difference in antimicrobial use was calculated by DOT per 1000 patient-days (DOT/1000PD), as an adjusted rate of antimicrobial exposure. We also compared the antimicrobial use based on defined daily dose (DDD) and DDD per 1000 patientdays (DDD/1000PD) between the cases and the controls.

\section{Time at risk and comparisons of antimicrobial use}

For the cases with MDRAB, the time-at-risk was calculated as the number of days from the date of admission to the date of MDRAB detection. For the controls without MDRAB isolation during hospitalization, the timeat-risk was calculated as for the cases starting on the date of admission. For the evaluation of clearance of MDRAB, the time-at-risk was the number of days between MDRAB isolation and MDRAB clearance. The adjusted rate of antimicrobial use (DOT/1000PD) was then calculated and compared between 1) the cases and the controls, and 2) before and after MDRAB isolation.

\section{Identification of multidrug-resistant Acinetobacter baumannii}

Identification of $A$. baumannii was performed by Matrix-Assisted Laser Desorption-Time Of Flight (MALDITOF) mass spectrometry (MS). Susceptibility to all tested antibiotics except tigecycline was determined according to the Clinical and Laboratory Standards Institute (CLSI) interpretive criteria for disk diffusion method. Susceptibility to tigecycline was determined using the disk diffusion method with Mueller-Hinton agar (BD Microbiology Systems, Cockeysville, MD), with breakpoints at $\geqq 16 \mathrm{~mm}$ and $\leqq 12 \mathrm{~mm}$. MDRAB was defined as $A$. baumannii with full or intermediate resistance to amikacin, gentamicin, cefepime, ceftazidime, piperacillin, piperacillin-tazobactam, aztreonam, ciprofloxacin, and carbapenems [18].

\section{Statistical analysis}

We used a multistate model analyzing the process of acquisition and clearance of the $\operatorname{MDRAB}[25,26]$. The multistate model described the two states (MDRAB negative and MDRAB positive) of the patients and also allowed estimation of the transition rate (acquisition and clearance) between the two states. When MDRAB was isolated from a given patient's clinical specimens, the patient was considered to have transitioned from the negative state to the positive state (MDRAB acquisition). The multistate model used a transition intensity matrix to model the transition rate between the two states. Another detail of the multistate model included the assumption that each transition between states followed a "no memory" property that could be described as an exponential distribution. When analyzing the effect of covariates, the Cox proportional hazard regression model was used. The significance of a covariate was determined using the Wald test statistic, which was calculated as the difference in the transition probability between groups divided by the standard error (square root of the sum of the 2 variances). The effects of covariates on the transition rates were quantified as the hazard ratio (HR), with a ratio greater than 1 indicating a positive effect on the transition rate. Other continuous variables were compared using Student's t test or the Mann-Whitney U test. Binomial variables were compared using chi-squared or Fisher's exact test. A 2-tailed $p$ value $<0.05$ was considered statistically significant in all tests. All statistical analyses were performed with SAS software (V9.4; SAS Institute, Inc., Cary, NC, USA).

\section{Results}

MDRAB occurred in nine of forty-two patients during the study period $(21.4 \%)$. In most ( 8 of 9 ) of these instances, MDRAB was detected from wounds. In only one instance was MDRAB isolated from the tip of a removed central venous catheter (CVC). Compared to the control group, the case group had similar demographics, total body surface area (TBSA) affected by burn, and APACHE II score on admission. No comorbid illness was reported in either group of patients. The median day to MDRAB occurrence was 16 days. Acquisition of MDRAB was associated with higher use of carbapenem (652 DOT/1000PD vs. 385 DOT/1000PD, $P<0.001$ ), lower use of non-carbapenem beta-lactam (298 DOT/ $1000 \mathrm{PD}$ vs. $534 \mathrm{DOT} / 1000 \mathrm{PD}, P<0.001)$. There was no difference of overall antibiotic use between the case group and the control group (1929 DOT/1000PD vs. 1890 DOT/1000PD, $P=0.759$ ). Measurement of DOT was comparable with that of DDD (correlation coefficient $=0.94)($ Table 1$)$.

Antimicrobial use was compared before and after the acquisition of MDRAB in the case group (Table 2). The analysis excluded one patient because the MDRAB was isolated from a CVC tip and clearance could not be defined. Antimicrobial exposure differed significantly in 
Table 1 Demographics and antimicrobial use of the healthy young adults admitted due to a dust explosion with or without MDRAB occurrence

\begin{tabular}{|c|c|c|c|}
\hline & $\operatorname{MDRAB}(n=9)$ & No MDRAB $(n=33)$ & $P$ value \\
\hline Age, median year (IQR) & $19(18-21)$ & $21(19-24)$ & 0.275 \\
\hline Male, no. (\%) & $2(22)$ & $11(49)$ & 0.258 \\
\hline TBSA, \% & $50(40-60)$ & $45(30-58)$ & 0.460 \\
\hline APACHE II score (IQR) & $6(4-7)$ & $6(4-7)$ & 0.409 \\
\hline Inhalation injury & $2(22)$ & $14(44)$ & 0.442 \\
\hline Mechanical ventilation on admission, no. (\%) & $5(56)$ & $22(67)$ & 0.698 \\
\hline Mechanical ventilation at week 1, no. (\%) & $6(67)$ & $25(76)$ & 0.676 \\
\hline Mechanical ventilation at week 2, no. (\%) & $5(56)$ & $21(64)$ & 0.711 \\
\hline LOS before MDRAB occurrence, day (IQR) & $16(11-18)$ & NA & NA \\
\hline Hospital day, median day (IQR) & $93(48-112)$ & $53(34-79)$ & 0.018 \\
\hline Bacteremia, no. (\%) & $2(22)$ & $10(24)$ & 1.000 \\
\hline In-hospital mortality, no. (\%) & $0(0)$ & $2(6 \%)$ & 1.000 \\
\hline \multicolumn{4}{|l|}{ Antimicrobial exposure ${ }^{a}$} \\
\hline Time-at-risk, patient-days ${ }^{b}$ & 141 & 517 & \\
\hline All antibiotics, DOT (DOT/1000 patient-days) & $272(1929)$ & $977(1890)$ & 0.759 \\
\hline Carbapenem & $92(652)$ & $199(385)$ & $<0.001$ \\
\hline Non-carbapenem Beta-lactam & $42(298)$ & $276(534)$ & $<0.001$ \\
\hline Glycopeptide & $116(823)$ & $384(743)$ & 0.335 \\
\hline Miscellaneous & $22(156)$ & $118(228)$ & 0.093 \\
\hline Antifungal agent, DOT (DOT/1000 patient-days) & $51(361)$ & $153(296)$ & 0.218 \\
\hline All antibiotics, DDD (DDD/1000 patient-days) & $299(2120)$ & $1099(2126)$ & 0.975 \\
\hline Carbapenem & $91(642)$ & $187(362)$ & $<0.001$ \\
\hline Non-carbapenem Beta-lactam & $61(431)$ & $360(695)$ & $<0.001$ \\
\hline Glycopeptide & $126(893)$ & $427(826)$ & 0.435 \\
\hline Miscellaneous & $22(153)$ & $125(243)$ & 0.050 \\
\hline Antifungal agent, DDD (DDD/1000 patient-days) & $81(574)$ & $276(533)$ & 0.557 \\
\hline
\end{tabular}

Data are expressed as median and interquartile range or number (\%) of patients unless otherwise indicated

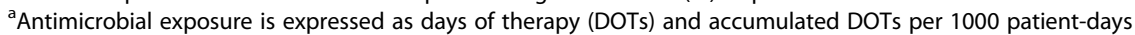

${ }^{b}$ Time-at-risk for the controls was calculated as for the cases starting on the date of admission in a 1:3 or 1:4 case-control match. The time-at-risk of those controls were matched with that of the cases, if possible

$A P A C H E$ Acute Physiology and Chronic Health Evaluation, $D D D$ defined daily dose, DOT days of therapy, IQR interquartile range, $L O S$ length of stay, MDRAB multidrug-resistant Acinetobacter baumannii, NA not applicable, TBSA total body surface area

Table 2 Comparison of antimicrobial use among the eight ${ }^{a}$ MDRAB cases before and after the occurrence of MDRAB

\begin{tabular}{llll}
\hline & Before & After & $P$ value \\
\hline Time-at-risk, patient-days $^{\mathrm{b}}$ & 130 & 98 & \\
All antibiotics, DOT (DOT/1000 patient-days) & $1923(1685-2161)$ & $2286(1986-2585)$ & 0.113 \\
$\quad$ Carbapenem & $708(563-852)$ & $469(334-605)$ & 0.003 \\
$\quad$ Non-carbapenem Beta-lactam & $246(161-331)$ & $612(457-767)$ & $<0.001$ \\
Glycopeptide & $846(688-1004)$ & $939(747-1131)$ & 0.424 \\
$\quad$ Miscellaneous & $123(63-183)$ & $265(163-367)$ & 0.016 \\
Antifungal agent & $392(285-500)$ & $765(592-939)$ & $<0.001$ \\
\hline
\end{tabular}

${ }^{a}$ One of the nine patients was excluded from the analysis. For this patient, only one MDRAB isolate was discovered from a removed central venous catheter (CVC) and there was no further MDRAB isolation from CVC available during study period. Thus, clearance of MDRAB was not defined and the analysis did not include the patient

${ }^{\mathrm{b}}$ Time-at-risk before MDRAB occurrence was the time from admission to MDRAB isolation. Time-at-risk after MDRAB occurrence was the time from MDRAB isolation to MDRAB clearance

Data are expressed as days of therapy (DOTs) and accumulated DOTs per 1000 patient-days

$D O T$ days of therapy, MDRAB multidrug-resistant Acinetobacter baumannii 
carbapenem, non-carbapenem beta-lactam antibiotics, and miscellaneous antibiotics. There was no difference in use of glycopeptide. Clearance of MDRAB was associated with fewer carbapenem use after adjusted with time-atrisk (469 DOT/1000PD vs. $708 \mathrm{DOT} / 1000 \mathrm{PD}, P=0.003$ ). Characteristics, outcomes, as well as detailed antimicrobial use before and after the isolation of MDRAB in the cases $(N=9)$ were compared. Four of whom have stopped carbapenem and received ceftazidime after the MDRAB occurrence. Three of whom have continued carbapenem use. Two of whom have continued non-carbapenem betalactam use (Table 3).

In the null model without considering any clinical correlates, the rate of transition from MDRAB negative to MDRAB positive (MDRAB acquisition) was 0.005 , while the rate of transition from MDRAB positive to MDRAB negative (MDRAB clearance) was 0.01 . The risk of acquiring MDRAB sharply increased 3 days after admission (Fig. 1). Antibiotic exposure had a significant effect on the transition between MDRAB negative and MDRAB positive (Table 4). In univariate analysis, carbapenem significantly affected the rate of MDRAB acquisition. Every additional carbapenem DOT increased the hazard of acquiring MDRAB by $9 \%$ (HR, 1.09; $95 \%$ CI 1.02-1.16; $P=0.007$ ) (Table 4). Glycopeptide, non-carbapenem beta-lactam, and antifungal agents showed no significant effect on MDRAB acquisition. Regarding clearance of MDRAB, every additional non-carbapenem beta- lactam DOT increased the protection of clearing MDRAB by $19 \%$ (HR, 1.19; 95\% CI 1.05-1.34; $P=0.024$ ) (Table 4). Carbapenem, glycopeptide, and antifungal agents showed no significant effect on MDRAB clearance. In multivariate analysis, carbapenem retained a positive effect on MDRAB acquisition (HR, 1.08; 95\% CI 1.01-1.16; $P=0.020)$ as did non-carbapenem beta-lactam on MDRAB clearance $(\mathrm{HR}, 1.25$; 95\% CI 1.07-1.46; $P=0.004)$ (Table 4).

\section{Discussion}

We conducted this study to examine the association between carbapenem and MDRAB in an extremely homogeneous group of previously healthy young burn patients. Our results show that antibiotic exposure was related to both the acquisition and clearance of MDRAB. In particularly, MDRAB clearance was associated with restricted use of carbapenem. Each additional DOT of carbapenem increased the risk of MDRAB acquisition, and each additional DOT of non-carbapenem betalactam increased the chance of MDRAB clearance. Our findings highlight the impact of antimicrobial use on the occurrence of drug-resistant pathogens, suggesting that the early discontinuation of carbapenem use may help to control the emergence of MDRAB.

Munoz-Price et al. [27] reported that every additional defined daily dose (DDD) of carbapenem increased the risk of carbapenem-resistant A. baumannii colonization (among patients initially found to be non-colonized) by

Table 3 Characteristics of the nine patients with multi-drug resistant Acinetobacter baumannii isolation

\begin{tabular}{|c|c|c|c|c|c|c|c|c|}
\hline No. & Source & $\begin{array}{l}\text { Concurrent } \\
\text { bacteremia }\end{array}$ & $\begin{array}{l}\text { APACHE ॥ } \\
\text { score }\end{array}$ & $\begin{array}{l}\text { TBSA } \\
(\%)\end{array}$ & $\begin{array}{l}\text { LOS before } \\
\text { MDRAB } \\
\text { occurrence }\end{array}$ & $\begin{array}{l}\text { Days from MDRAB } \\
\text { occurrence to } \\
\text { clearance }\end{array}$ & $\begin{array}{l}\text { Antimicrobial use } \\
\text { before MDRAB } \\
\text { isolation }\end{array}$ & $\begin{array}{l}\text { Antimicrobial use } \\
\text { after MDRAB isolation }\end{array}$ \\
\hline 1 & Wound & no & 6 & 40 & 16 & 10 & $\begin{array}{l}\text { Teicoplanin, } \\
\text { imipenem }\end{array}$ & Teicoplanin, ceftazidime \\
\hline 2 & Wound & no & 4 & 60 & 15 & 14 & $\begin{array}{l}\text { Teicoplanin, } \\
\text { imipenem }\end{array}$ & Teicoplanin, ceftazidime \\
\hline 3 & Wound & no & 7 & 40 & 22 & 9 & $\begin{array}{l}\text { Teicoplanin, } \\
\text { imipenem }\end{array}$ & Teicoplanin, meropenem \\
\hline 4 & CVC & no & 4 & 35 & 11 & NA & $\begin{array}{l}\text { Teicoplanin, } \\
\text { ceftazidime }\end{array}$ & Teicoplanin, ceftazidime \\
\hline 5 & Wound & $\begin{array}{l}\text { Klebsiella } \\
\text { pneumoniae } \\
\text { Staphylococcus haemolyticus }\end{array}$ & 9 & 55 & 11 & 21 & $\begin{array}{l}\text { Teicoplanin, } \\
\text { imipenem }\end{array}$ & Daptomycin, imipenem \\
\hline 6 & Wound & no & 6 & 60 & 26 & 10 & $\begin{array}{l}\text { Teicoplanin, } \\
\text { ceftazidime }\end{array}$ & Cefepime \\
\hline 7 & Wound & $\begin{array}{l}\text { Acinetobacter pittii } \\
\text { Staphylococcus haemolyticus }\end{array}$ & 13 & 80 & 16 & 14 & $\begin{array}{l}\text { Teicoplanin, } \\
\text { imipenem }\end{array}$ & $\begin{array}{l}\text { Teicoplanin + impenem } \\
+ \text { colistin, teicoplanin }+ \\
\text { cefaperazone/sulbactam }\end{array}$ \\
\hline 8 & Wound & no & 6 & 50 & 18 & 10 & $\begin{array}{l}\text { Teicoplanin, } \\
\text { imipenem }\end{array}$ & Teicoplanin + ceftazidime \\
\hline 9 & Wound & no & 4 & 40 & 6 & 10 & $\begin{array}{l}\text { Teicoplanin, } \\
\text { ceftazidime }\end{array}$ & $\begin{array}{l}\text { Teicoplanin + cefaperazone/ } \\
\text { sulbactam }\end{array}$ \\
\hline
\end{tabular}

MDRAB was cleared before discharge for all the patients except one person (No. 4) who had a removed central venous catheter (CVC) with MDRAB detection. All the cases survived and were discharged from the hospital uneventfully

CVC central venous catheter, LOS length of stay, MDRAB multi-drug resistant Acinetobacter baumannii, NA not applicable, TBSA total body surface area 


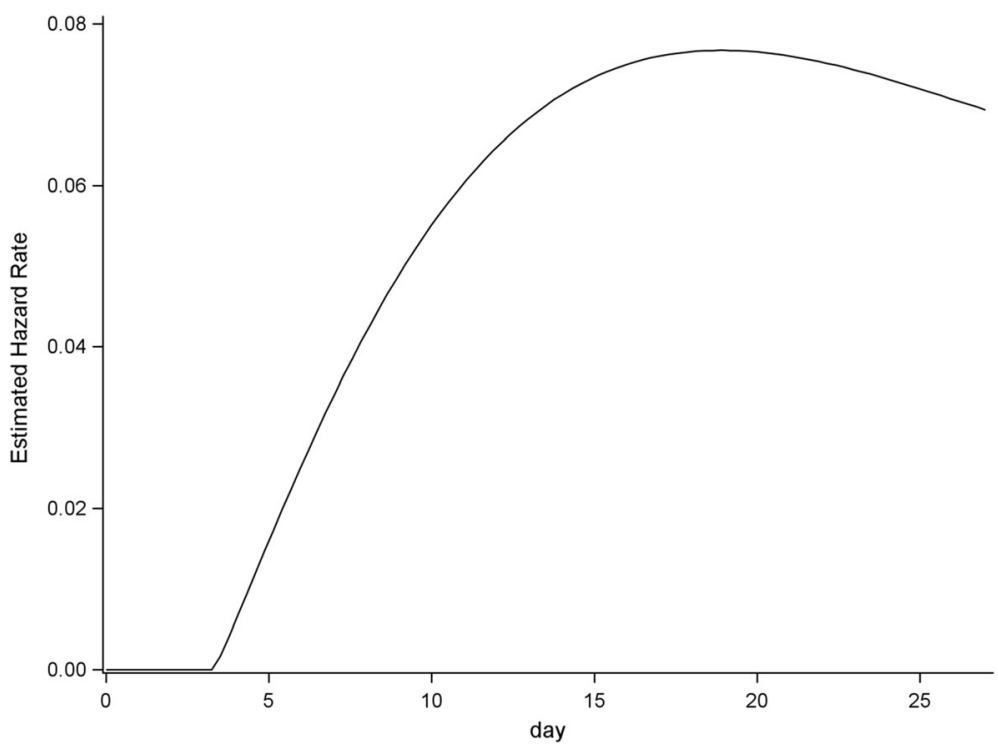

Fig. 1 The overall hazard rate of acquiring MDRAB in the first 28 days after admission among the study population

$5.1 \%$. In the group of previously healthy young burn patients, we demonstrated that carbapenem was a timedependent variable for MDRAB acquisition, and every additional DOT increased the risk of MDRAB acquisition by $8 \%$. Both the studies explored the effect of carbapenem exposure as a continuous variable to further understand the association between time-dependent antibiotic exposure and acquisition of MDRAB. However, our cohort is uniquely positioned to evaluate the impact of antibiotic exposure on the occurrence of MDRAB infection/colonization, given that our patients were free of underlying conditions and were admitted at the same time due to the dust explosion; the results of these experiments provided additional data to facilitate the optimization of antibiotic prescription by clinicians.
The importance of detection of carbapenem-resistant $A$. baumannii colonization lies in the high predictive value with regard to the subsequent development of carbapenemresistant A. baumannii infection [28]. Notably, infection by carbapenem-resistant A. baumannii is associated with a mortality rate of approximately $52 \%$, compared with a $19 \%$ mortality rate observed following infection with carbapenem-susceptible A. baumannii [29]. Our findings support the short-term impact of an ASP on the clearance of drug-resistant pathogens; the early discontinued use of carbapenem may help to control an MDRAB outbreak. In the long term, carbapenem use may increase following the implementation of ASP due to the ballooning effect [15]. Carbapenems, which exhibit broad-spectrum activity against bacteria, may

Table 4 Hazard ratios for the acquisition and clearance of MDRAB

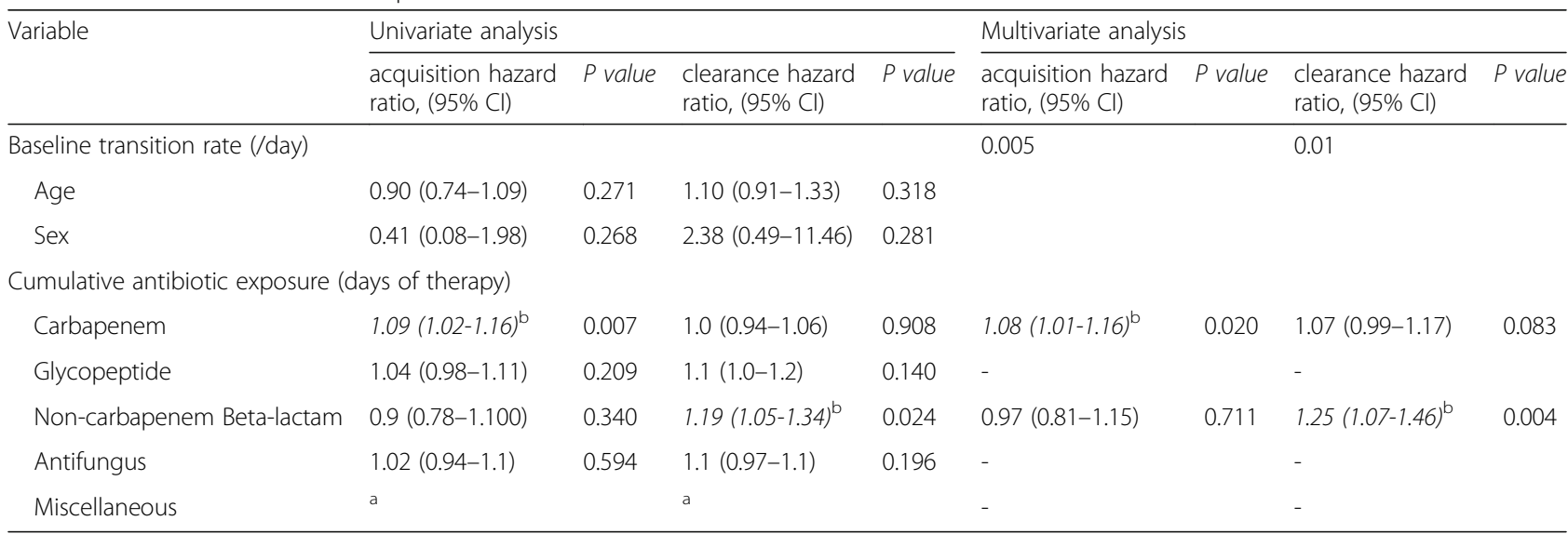

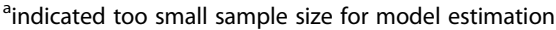

${ }^{\mathrm{b}}$ Hazard ratio with statistical significance 
impose collateral damage and perturb the human indigenous microbiota [30]. Halting carbapenem use may be helpful for reframing and restoring the human microbiome, which may combat MDRAB, and for precluding the selection of resistant strains from a population of susceptible bacteria. Our results also suggest that the competitive advantage of $A$. baumannii in the microbial ecology may solely reflect this organism's resistance to antimicrobial agents under selective pressure.

There has been a report that beta-lactam was a risk factor for acquisition of MDRAB [31]. Furthermore, differing from our findings, the usage of carbapenem tended to correlate to clearance of MDRAB in the multivariable analysis with borderline significance [31]. One possible reason is that our patient group was comprised of a cohort of relatively young burn victims without comorbid illnesses. There were few potential confounding variables. Most of the case patients (7/9) were cleared from MDRAB without antimicrobial therapy directed against MDRAB (Table 3). Still, the patients received other antibiotics due to critically ill conditions and burn injury. There were two of the nine cases who received specific treatment for MDRAB (Case No. 7 and case No. 9 in Table 3). Despite limited number of cases, our findings may imply the possibility of spontaneously clearance of MDRAB in immune competent hosts. It might be partly caused by less selective pressure and the clearance could occur without pharmacological intervention.

Both DDD and DOT are standardized methods for measurement of antibiotic use [32]. We chose DOT divided by time-at-risk (i.e., accumulated person days) to compare the rate of antimicrobial use between the case and the control group. As the measure of antimicrobial use that best predicts the prevalence of antimicrobial resistance has not yet been defined [33], one advantage of DOT is that it is not affected by changes in dosing regimen [21]. Moreover, DOT could be more helpful in comparing the use of different classes of antimicrobials within a given institution and indicate timely antimicrobial usage in a particular patient [21]. Because the clinicians might adjust the antimicrobial regimens promptly when treating critically ill patients, we think that measurement of the DOTs would be a better reflection of clinical scenario and usage of antimicrobial agents.

Our study does have some limitations. Due to this study's retrospective nature and single-center design, our results may be not applicable in other settings or hospitals. There were more than 400 injuries due to the dust explosion and only 42 of the them who were admitted to the ICUs of the hospital were included. This has implications for possible selection bias. However, our study population consisted of young adults who were previously healthy and admitted as a concurrent group following a single event (the dust explosion). The patients were admitted to the hospital at the same time between June 27, 2015 and July 1, 2015. We acknowledged that medical procedures and environmental factors played important roles in the process of MDRAB colonization. The integrated and intensive care provided by the hospital at that time maximally eliminated the confounding factors that were measurable [23]. Hence, the results are expected to be relevant and may contribute to clinical decision-making. Another potential drawback is that we did not investigate the indications of antimicrobial use in our analysis. However, a well-established integrated ASP has been implemented in the hospital for a decade; each prescription was carefully reviewed daily by both pharmacists and infection diseases specialists [10, 12]. As a result, decisions regarding antimicrobial use were attributable to responsible clinicians; appropriate use of antimicrobial agents could be expected in the study. The definition of MDRAB clearance among the patients with prior MDRAB isolation may be concerned. We defined MDRAB clearance according to the hospital's policy, which requires surveillance cultures to be obtained within 7 days of the previous culture. By definition, eight of nine MDRAB-positive patients were clear from MDRAB. Biases were minimized, given that the ASP were applied regularly and consistently across the hospital. A further limitation of our study was that we did not perform contemporaneous environmental surveillances in either of the two ICUs. This precluded the analysis of interactions between the patients and their hospital environment. Finally, we did not discern between infection and colonization status in the study. Yet the conclusion may be valid in either infection or colonization status.

\section{Conclusions}

In conclusion, our results suggest that antibiotic exposure is associated with both acquisition and clearance of MDRAB in this homogeneous population of previously healthy, critically ill young burn patients. Our findings reinforce the utility of an ASP in reducing the occurrence of multidrug-resistant pathogens, and highlight the appropriateness of early discontinuation of carbapenem when the use of carbapenem is inevitable.

\section{Abbreviations \\ ASP: antibiotic stewardship program; Cl: Confidence interval; CVC: central venous catheter; DDD: Defined daily dose; DOT: Day of therapy; HR: Hazard ratio; ICU: Intensive care unit; MDRAB: Multidrug-resistant Acinetobacter baumannii; TBSA: total body surface area}

\section{Acknowledgments}

We are grateful to the infection control group and the members of the laboratory medicine for their excellent work and support in the hospital.

\section{Funding}

This work was supported partly by grants from the Chang Gung Memorial Hospital (CORPG3F0171). No funding sources had any role in the design or 
conduct of the study; collection, management, analysis, or interpretation of the data; or preparation, review, or approval of the manuscript.

\section{Availability of data and materials}

Complete data will be provided upon request by the corresponding author (chingtaihuang@gmail.com).

\section{Authors' contributions}

SSS, PYH, YJH, and CTH designed the study, collected and analysed the data, and wrote the article. SSS and PYH were responsible for the integrity data and performed statistical analysis together with SPL. JJY, TSW, SSC, MHC provided intellectual input and joined the discussion. TPL and TLW were responsible for the microbiological identification and provided intellectual input. All authors have read and approved the final article.

\section{Ethics approval and consent to participate}

This retrospective study was approved by institutional review boards of Chang Gung Memorial Hospital (Number: 1049356B). The ethics committee granted a waiver for informed consent to be obtained.

\section{Consent for publication}

Not applicable

\section{Competing interests}

The authors declare that they have no competing interests.

\section{Publisher's Note}

Springer Nature remains neutral with regard to jurisdictional claims in published maps and institutional affiliations.

\section{Author details}

'Division of Infectious Diseases, Department of Medicine, Chang Gung Memorial Hospital and Chang Gung University, 5 Fu-Shin St., Kweishan, 333 Taoyuan, Taiwan. ${ }^{2}$ Infection Control Committee, Chang Gung Memorial Hospital and Chang Gung University, Taoyuan, Taiwan. ${ }^{3}$ Division of Biostatistics, Graduate Institute of Epidemiology and Preventive Medicine, National Taiwan University, Taipei, Taiwan. ${ }^{4}$ Department of Anesthesiology, Taipei Veterans General Hospital and National Yang-Ming University School of Medicine, Taipei, Taiwan. ${ }^{5}$ Department of Laboratory Medicine, Chang Gung Memorial Hospital and Chang Gung University, Taoyuan, Taiwan. ${ }^{6}$ Department of Plastic and Reconstructive Surgery, Chang Gung Memorial Hospital and Chang Gung University, Taoyuan, Taiwan. ${ }^{7}$ Division of Pediatric Infectious Diseases, Department of Pediatrics, Chang Gung Children's Hospital, Chang Gung University College of Medicine, 5 Fu-Shin St., Kweishan, 333 Taoyuan, Taiwan.

\section{Received: 8 June 2017 Accepted: 15 August 2017}

Published online: 30 August 2017

\section{References}

1. Fournier PE, Richet $\mathrm{H}$. The epidemiology and control of Acinetobacter Baumannii in health care facilities. Clin Infect Dis. 2006;42:692-9.

2. Kempf M, Rolain JM. Emergence of resistance to carbapenems in Acinetobacter Baumannii in Europe: clinical impact and therapeutic options. Int J Antimicrob Agents. 2012;39:105-14.

3. Jawad A, Seifert H, Snelling AM, Heritage J, Hawkey PM. Survival of Acinetobacter Baumannii on dry surfaces: comparison of outbreak and sporadic isolates. J Clin Microbiol. 1998;36:1938-41.

4. Boucher HW, Talbot GH, Bradley JS, Edwards JE, Gilbert D, Rice LB, Scheld M, Spellberg B, Bartlett J. Bad bugs, no drugs: no ESKAPE! An update from the Infectious Diseases Society of America. Clin Infect Dis. 2009;48:1-12.

5. Pogue JM, Mann T, Barber KE, Kaye KS. Carbapenem-resistant Acinetobacter Baumannii: epidemiology, surveillance and management. Expert Rev AntiInfect Ther. 2013:11:383-93.

6. Kim T, Chong YP, Park SY, Joen MH, Choo EJ, Chung JW, Lee HK, Moon C, Kim DM, Peck KR, Kim YS. Risk factors for hospital-acquired pneumonia caused by carbapenem-resistant gram-negative bacteria in critically ill patients: a multicenter study in Korea. Diagn Microbiol Infect Dis. 2014;78:457-61

7. Gray AP, Allard R, Pare R, Tannenbaum T, Lefebvre B, Levesque S, Mulvey M, Maalouf L, Perna S, Longtin Y. Management of a hospital outbreak of extensively drug-resistant Acinetobacter Baumannii using a multimodal intervention including daily chlorhexidine baths. J Hosp Infect. 2016;93:29-34.

8. Gavalda L, Soriano AM, Camara J, Gasull R, Arch O, Ferrer M, Shaw E, Granada RM, Dominquez MA, Pujol M. Control of endemic extensively drug-resistant Acinetobacter Baumannii with a cohorting policy and cleaning procedures based on the 1 room, 1 wipe approach. Am J Infect Contro. 2016:44:520-4.

9. Chinese XDR Consensus Working Group, Guan X, He L, Hu B, Hu J, Huang $X$, et al. Laboratory diagnosis, clinical management and infection control of the infections caused by extensively drug-resistant Gramnegative bacilli: a Chinese consensus statement. Clin Microbiol Infect. 2016;22:S15-25.

10. Molter G, Seifert H, Mandraka F, Kasper G, Weidmann B, Hornei B, Öhler M, Schwimmbeck P, Kröschel P, Higgins PG, Reuter S. Outbreak of carbapenem-resistant Acinetobacter Baumannii in the intensive care unit: a multi-level strategic management approach. J Hosp Infect. 2016;92:194-8.

11. Martin C, Ofotokun I, Rapp R, Empey K, Armitstead J, Pomeroy C, Hoven A, Evans M. Results of an antimicrobial control program at a university hospital. Am J Health Syst Pharm 1 2005;62:732-738.

12. Kaki $R$, Elligsen $M$, Walker $S$, Simor $A$, Palmay $L$, Daneman N. Impact of antimicrobial stewardship in critical care: a systematic review. J Antimicrob Chemother. 2011;66:1223-30.

13. Wang HY, Chiu CH, Huang CT, Cheng CW, Lin YJ, Hsu YJ, Chen CH, Deng ST, Leu HS. Blood culture-guided de-escalation of empirical antimicrobial regimen for critical patients in an online antimicrobial stewardship programme. Int J Antimicrob Agents. 2014;44:520-7.

14. Lai CC, Shi ZY, Chen YH, Wang FD. Effects of various antimicrobial stewardship programs on antimicrobial usage and resistance among common gram-negative bacilli causing health care-associated infections: a multicenter comparison. J Microbiol Immunol Infect. 2016;49:74-82.

15. Chan YY, Lin TY, Huang CT, Deng ST, Wu TL, Leu HS, Chiu CH. Implementation and outcomes of a hospital-wide computerised antimicrobial stewardship programme in a large medical centre in Taiwan. Int J Antimicrob Agents Dec. 2011;38:486-92.

16. Sun L, Klein EY, Laxminarayan R. Seasonality and temporal correlation between community antibiotic use and resistance in the United States. Clin Infect Dis. 2012:55:687-94.

17. Kallel H, Mahjoubi F, Dammak H, Bahloul M, Hamida CB, Chelly H, Rekik N, Hammami A, Bouaziz M. Correlation between antibiotic use and changes in susceptibility patterns of Pseudomonas Aeruginosa in a medical-surgical intensive care unit. Indian J Crit Care Med. 2008;12:18-23.

18. Ye JJ, Huang CT, Shie SS, Huang PY, Su LH, Chiu CH, Leu HS, Chiang PC. Multidrug resistant Acinetobacter Baumannii: risk factors for appearance of imipenem resistant strains on patients formerly with susceptible strains. PLoS One. 2010;5:e9947.

19. Inchai J, Liwsrisakun C, Theerakittikul T, Chaiwarith R, Khositsakulchai W, Pothirat C. Risk factors of multidrug-resistant, extensively drug-resistant and pandrug-resistant Acinetobacter Baumannii ventilator-associated pneumonia in a medical intensive care unit of University Hospital in Thailand. J Infect Chemother. 2015:21:570-4.

20. Liu Q, Li W, Du X, Li W, Zhong T, Tang Y, Feng Y, Tao C, Xie Y. Risk and prognostic factors for multidrug-resistant Acinetobacter Baumannii complex bacteremia: a retrospective study in a tertiary Hospital of West China. PLoS One. 2015;10:e0130701.

21. Doron S, Davidson LE. Antimicrobial stewardship. Mayo Clin Proc. 2011:86:1113-23.

22. Reiner M. Taiwan's emergency medical care-learning from disaster. 2015; http://topics.amcham.com.tw/2015/08/taiwans-emergency-medical-carelearning-from-disaster/. Accessed November 18, 2016.

23. Cheng MH, Mathews AL, Chuang SS, Lark ME, Hsiao YC, Ng CJ, Chung KC. Management of the Formosa Color Dust Explosion: lessons learned from the treatment of 49 mass burn casualty patients at Chang gung memorial hospital. Plast Reconstr Surg. 2016;137:1900-8.

24. Polk RE, Fox C, Mahoney A, Letcavage J, MacDougall C. Measurement of adult antibacterial drug use in 130 US hospitals: comparison of defined daily dose and days of therapy. Clin Infect Dis. 2007;44:664-70.

25. Chen TH, Kuo HS, Yen MF, Lai MS, Tabar L, Duffy SW. Estimation of sojourn time in chronic disease screening without data on interval cases. Biometrics. 2000:56:167-72.

26. Hui-Min W, Ming-Fang Y, Chen TH. SAS macro program for nonhomogeneous Markov process in modeling multi-state disease progression. Comput Methods Prog Biomed. 2004;75:95-105. 
27. Munoz-Price LS, Rosa R, Castro JG, Laowansiri P, Latibeaudiere R, Namias N, Tarima S. Evaluating the impact of antibiotic exposures as time-dependent variables on the Acquisition of Carbapenem-Resistant Acinetobacter Baumannii. Crit Care Med. 2016:44:e949-56.

28. Latibeaudiere R, Rosa R, Laowansiri P, Arheart K, Namias N, Munoz-Price LS Surveillance cultures growing carbapenem-resistant Acinetobacter Baumannii predict the development of clinical infections: a retrospective cohort study. Clin Infect Dis. 2015;60:415-22

29. Jamulitrat S, Arunpan P, Phainuphong P. Attributable mortality of imipenem-resistant nosocomial Acinetobacter Baumannii bloodstream infection. J Med Assoc Thail. 2009:92:413-9.

30. Kager L, Brismar B, Malmborg AS, Nord CE. Imipenem concentrations in colorectal surgery and impact on the colonic microflora. Antimicrob Agents Chemother. 1989;33:204-8.

31. Tsai HT, Wang JT, Chen CJ, Chang SC. Association between antibiotic usage and subsequent colonization or infection of extensive drug-resistant Acinetobacter Baumannii: a matched case-control study in intensive care units. Diagn Microbiol Infect Dis. 2008;62:298-305.

32. Barlam TF, Cosgrove SE, Abbo LM, MacDougall C, Schuetz AN, Septimus EJ et al. Implementing an antibiotic stewardship program: guidelines by the Infectious Diseases Society of America and the Society for Healthcare Epidemiology of America. Clin Infect Dis. 2016;62:e51-77.

33. Fortin E, Fontela PS, Manges AR, Platt RW, Buckeridge DL, Quach C. Measuring antimicrobial use in hospitalized patients: a systematic review of available measures applicable to paediatrics. J Antimicrob Chemother. 2014;69:1447-56.

\section{Submit your next manuscript to BioMed Central and we will help you at every step:}

- We accept pre-submission inquiries

- Our selector tool helps you to find the most relevant journal

- We provide round the clock customer support

- Convenient online submission

- Thorough peer review

- Inclusion in PubMed and all major indexing services

- Maximum visibility for your research

Submit your manuscript at www.biomedcentral.com/submit 